\title{
Photo-oxidative tuning of individual and coupled GaAs photonic crystal cavities
}

\author{
Alexander Y. Piggott, ${ }^{1, *}$ Konstantinos G. Lagoudakis, ${ }^{1}$ \\ Tomas Sarmiento, ${ }^{1}$ Michal Bajcsy, ${ }^{1,2}$ Gary Shambat, ${ }^{1}$ \\ and Jelena Vučković ${ }^{1}$ \\ ${ }^{1}$ E. L. Ginzton Laboratory, Stanford University, California, USA \\ ${ }^{2}$ Institute for Quantum Computing, University of Waterloo, Ontario, Canada \\ *piggott@stanford.edu
}

\begin{abstract}
We demonstrate a photo-induced oxidation technique for tuning GaAs photonic crystal cavities using a low-power $390 \mathrm{~nm}$ pulsed laser. The laser oxidizes a small $(<1 \mu \mathrm{m})$ diameter spot, reducing the local index of refraction and blueshifting the cavity. The tuning progress can be actively monitored in real time. We also demonstrate tuning an individual cavity within a pair of proximity-coupled cavities, showing that this method can be used to tune individual cavities in a cavity network, with applications in quantum simulations and quantum computing.
\end{abstract}

(C) 2014 Optical Society of America

OCIS codes: (140.3945) Microcavities; (140.3948) Microcavity devices; (160.5298) Photonic crystals.

\section{References and links}

1. J. Vučković, M. Lončar, H. Mabuchi, and A. Scherer, "Design of photonic crystal microcavities for cavity QED," Phys. Rev. E 65, 016608 (2001).

2. T. Yoshie, A. Scherer, J. Hendrickson, G. Khitrova, H. M. Gibbs, G. Rupper, C. Ell, O. B. Shchekin, and D. G. Deppe, "Vacuum rabi splitting with a single quantum dot in a photonic crystal nanocavity," Nature 432, 200-203 (2004).

3. D. Englund, A. Faraon, I. Fushman, N. Stoltz, P. Petroff, and J. Vučković, "Controlling cavity reflectivity with a single quantum dot," Nature 450, 857-861 (2007).

4. Y. Akahane, T. Asano, B.-S. Song, and S. Noda, "High-Q photonic nanocavity in a two-dimensional photonic crystal," Nature 425, 944-947 (2003).

5. T. C. H. Liew and V. Savona, "Single photons from coupled quantum modes," Phys. Rev. Lett. 104, 183601 (2010).

6. M. Bamba, A. Imamoğlu, I. Carusotto, and C. Ciuti, "Origin of strong photon antibunching in weakly nonlinear photonic molecules,” Phys. Rev. A 83, 021802 (2011).

7. A. Majumdar, M. Bajcsy, A. Rundquist, and J. Vučković, "Loss-enabled sub-poissonian light generation in a bimodal nanocavity," Phys. Rev. Lett. 108, 183601 (2012)

8. A. D. Greentree, C. Tahan, J. H. Cole, and L. C. L. Hollenberg, "Quantum phase transitions of light," Nat. Phys. 2, 856-861 (2006).

9. A. Majumdar, A. Rundquist, M. Bajcsy, and J. Vučković, "Cavity quantum electrodynamics with a single quantum dot coupled to a photonic molecule," Phys. Rev. B 86, 045315 (2012).

10. J. Kerckhoff, H. I. Nurdin, D. S. Pavlichin, and H. Mabuchi, "Designing quantum memories with embedded control: Photonic circuits for autonomous quantum error correction," Phys. Rev. Lett. 105, 040502 (2010),

11. K. Hennessy, A. Badolato, A. Tamboli, P. M. Petroff, E. Hua, M. Atatüre, J. Dreiser, and A. Imamoğlu, "Tuning photonic crystal nanocavity modes by wet chemical digital etching," Appl. Phys. Lett. 87, 021108 (2005).

12. N. W. L. Speijcken, M. A. Dndar, A. C. Bedoya, C. Monat, C. Grillet, P. Domachuk, R. Nötzel, B. J. Eggleton, and R. W. van der Heijden, "In situ optofluidic control of reconfigurable photonic crystal cavities," Appl. Phys. Lett. 100, 261107 (2012).

13. S. Vignolini, F. Riboli, D. S. Wiersma, L. Balet, L. H. Li, M. Francardi, A. Gerardino, A. Fiore, M. Gurioli, and F. Intonti, "Nanofluidic control of coupled photonic crystal resonators," Appl. Phys. Lett. 96, 141114 (2010). 
14. T. Cai, R. Bose, G. S. Solomon, and E. Waks, "Controlled coupling of photonic crystal cavities using photochromic tuning," Appl. Phys. Lett. 102, 141118 (2013).

15. A. Faraon, D. Englund, D. Bulla, B. Luther-Davies, Benjamin, J. Eggleton, N. Stoltz, P. Petroff, and J. Vučković, "Local tuning of photonic crystal cavities using chalcogenide glasses," Appl. Phys. Lett. 92, 043123 (2008).

16. H. S. Lee, S. Kiravittaya, S. Kumar, J. D. Plumhof, L. Balet, L. H. Li, M. Francardi, A. Gerardino, A. Fiore, A. Rastelli, and O. G. Schmidt, "Local tuning of photonic crystal nanocavity modes by laser-assisted oxidation," Appl. Phys. Lett. 95, 191109 (2009).

17. K. Hennessy, C. Högerle, E. Hu, A. Badolato, and A. Imamolu, "Tuning photonic nanocavities by atomic force microscope nano-oxidation," Appl. Phys. Lett. 89, 041118 (2006).

18. F. Intonti, N. Caselli, S. Vignolini, F. Riboli, S. Kumar, A. Rastelli, O. G. Schmidt, M. Francardi, A. Gerardino, L. Balet, L. H. Li, A. Fiore, and M. Gurioli, "Mode tuning of photonic crystal nanocavities by photoinduced non-thermal oxidation,” Appl. Phys. Lett. 100, 033116 (2012).

19. I. J. Luxmoore, E. D. Ahmadi, B. J. Luxmoore, N. A. Wasley, A. I. Tartakovskii, M. Hugues, M. S. Skolnick, and A. M. Fox, "Restoring mode degeneracy in H1 photonic crystal cavities by uniaxial strain tuning," Appl. Phys. Lett. 100, 121116 (2012).

20. Y. Gong, B. Ellis, G. Shambat, T. Sarmiento, J. S. Harris, and J. Vučković, "Nanobeam photonic crystal cavity quantum dot laser,' Opt. Express 18, 8781-8789 (2010).

21. B. Ellis, M. A. Mayer, G. Shambat, T. Sarmiento, J. Harris, E. E. Haller, and J. Vučković, "Ultralow-threshold electrically pumped quantum-dot photonic-crystal nanocavity laser," Nat. Photon. 5, 297-300 (2011).

22. E. M. Purcell, "Spontaneous emission probabilities at radio frequencies," Phys. Rev. 69, 681 (1946)

23. H. A. Haus and W. Huang, "Coupled-mode theory," Proc. IEEE 79, 1505-1518 (1991).

24. C. Yu, D. Podlesnik, M. Schmidt, H. Gilgen, and R. M. Osgood Jr., "Ultraviolet-light-enhanced oxidation of gallium arsenide surfaces studied by x-ray photoelectron and auger electron spectroscopy," Chem. Phys. Lett. 130, 301-306 (1986).

25. C. F. Yu, M. T. Schmidt, D. V. Podlesnik, , and R. M. Osgood, "Wavelength dependence of optically induced oxidation of GaAs(100),” J. Vac. Sci. Technol. B 5, 1087-1091 (1987).

26. Z. Lu, M. T. Schmidt, D. V. Podlesnik, C. F. Yu, and R. M. Osgood, "Ultraviolet-light-induced oxide formation on GaAs surfaces," J. Chem. Phys. 93, 7951-7961 (1990).

27. J. Petykiewicz, G. Shambat, B. Ellis, and J. Vučković, "Electrical properties of GaAs photonic crystal cavity lateral p-i-n diodes," Appl. Phys. Lett. 101, 011104 (2012).

28. E. D. Palik, Handbook of Optical Constants of Solids (Elsevier, 1997).

29. S. M. Sze, Semiconductor Sensors (John Wiley, 1994).

\section{Introduction}

Photonic crystal cavities have been extensively used in studies of cavity quantumelectrodynamics (cQED) [1-3] owing to their ability to strongly confine light with high quality factors [4]. Extending this work, a wide range of quantum computing and quantum simulation proposals using coupled cavity networks have been put forward. These include sub-Poissonian light generation [5-7], the quantum simulation of exotic many-body systems [8,9], and quantum error correction [10]. GaAs is an excellent material for fabricating these coupled-cavity networks since thin membranes with embedded quantum dots can be grown epitaxially.

Managing cavity frequency variations caused by fabrication imperfections is a major challenge that must be overcome in order to implement systems based on coupled-cavity networks. Coupled cavity devices require the cavity resonances to be spectrally aligned with each other, but the resonant wavelength of identically designed photonic crystal cavities typically varies by several nanometers, even for devices only a few microns apart [7]. Thus, there has been considerable interest in the post-fabrication tuning of photonic crystal cavities.

A number of techniques for post-fabrication tuning of GaAs photonic crystal cavities have been demonstrated. These include wet etching [11], infiltration of water [12,13], deposition of photosensitive materials [14, 15], thermal oxidation [16], atomic-force microscope (AFM) oxidation [17], green laser photo-oxidation [18], and application of strain to the entire chip [19]. However, many of these techniques are not well localized and hence cannot be used to tune individual cavities in coupled cavity configurations, while others require the application of fluids or polymers, or the use of an AFM.

We describe a more convenient technique for tuning GaAs photonic crystal cavities using 
(a)

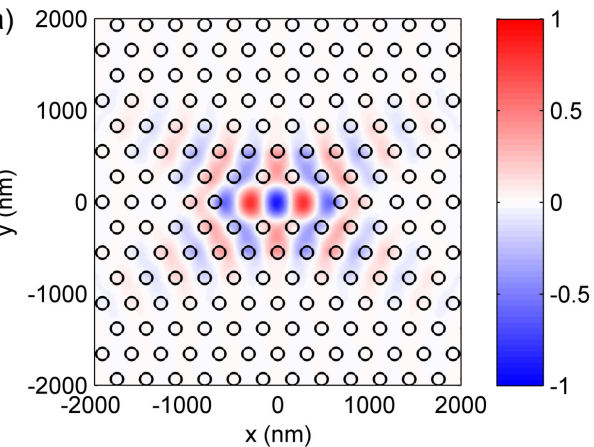

(b)

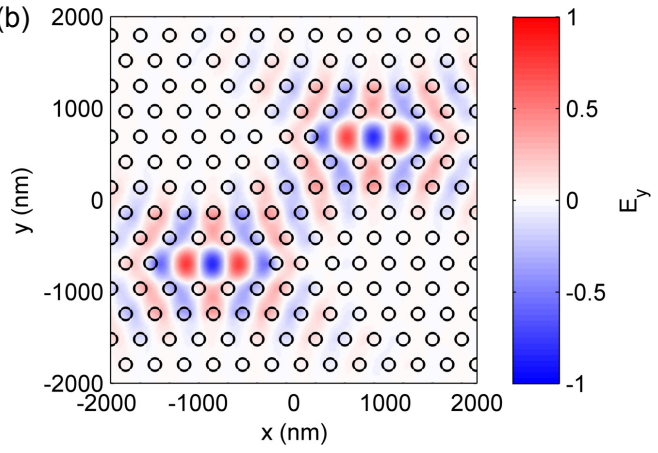

(c)

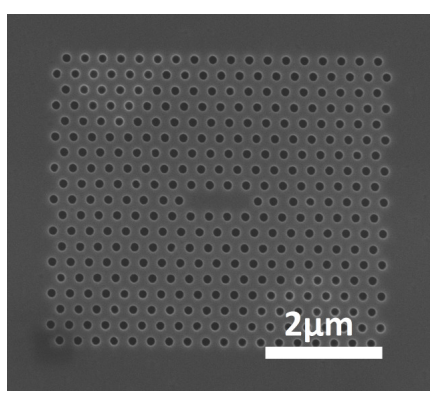

(d)

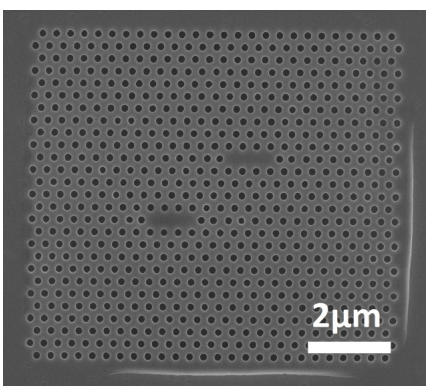

Fig. 1. (a,b) The transverse electric field $\left(E_{y}\right)$ distribution for the fundamental modes of a (a) single L3 cavity and (b) two coupled L3 cavities, calculated in a finite-difference time domain (FDTD) simulation. The coupled cavity supports both anti-symmetric and symmetric modes; here we have plotted the latter. The black circles indicate the locations of the holes in the photonic crystal membrane. (c,d) Scanning electron microscopy (SEM) images of a single (c) and coupled (d) GaAs L3 cavities, taken before performing any tuning.

$390 \mathrm{~nm}$ pulsed laser light to introduce photo-induced oxidation. The laser oxidizes a small $(<1 \mu \mathrm{m})$ diameter spot, lowering the local index of refraction and blueshifting the cavity. Our approach exploits the same physical mechanism used by Intonti et al. [18], which utilized a $532 \mathrm{~nm}$ laser at a relatively high power $(700 \mu \mathrm{W})$. By using a shorter-wavelength laser, we were able to reduce the tuning power by nearly two orders of magnitude while maintaining similar tuning rates, potentially enabling tuning of fragile structures such as nanobeam cavities [20]. Finally, to demonstrate the resolution and utility of our approach, we demonstrate tuning individual cavities in proximity-coupled pairs of cavities.

\section{Experimental technique}

The photonic crystal cavities used in this experiment were L3 cavities in a triangular photonic crystal lattice [4], with lattice constant $a=336 \mathrm{~nm}$ and design hole radius $r=0.212 a$. The fundamental mode for the L3 cavity calculated using finite-difference time-domain (FDTD) simulations is plotted in Fig. 1(a), and has a simulated quality factor of $\sim 4 \times 10^{4}$. We tested the proposed tuning mechanism on both individual cavities and pairs of proximity-coupled cavities. The coupled L3 defects were placed 5 lattice periods apart, with a spectral splitting of $1.2 \mathrm{~nm}$ calculated using FDTD. Scanning electron microscopy (SEM) images of these structures are shown in Figs. 1(c) and 1(d).

The photonic crystal cavities were fabricated from GaAs wafers grown using molecular beam

\#209768 - \$15.00 USD

(C) 2014 OSA 
epitaxy, as described in detail in previous work [21]. The structure was grown on a GaAs substrate by molecular beam epitaxy and consisted of a $1500 \mathrm{~nm} \mathrm{Al}_{0.8} \mathrm{Ga}_{0.2}$ As sacrificial layer and a $220 \mathrm{~nm}$ GaAs membrane. The GaAs membrane contained 3 layers of high-density InAs quantum dots $\left(300 \mathrm{dots} / \mu \mathrm{m}^{2}\right)$ emitting at $\sim 1300 \mathrm{~nm}$. The photonic crystal cavities were fabricated using electron-beam lithography, inductively-coupled plasma reactive-ion etching (ICP-RIE), and a final HF acid undercutting step, as described previously [3].

The tuning was performed in a custom confocal microscopy setup coupled to a grating spectrometer with an InGaAs linear photodiode array. A Carl Zeiss LD-Plan-Neofluar $63 x / 0.75$ Korr was used as the microscope objective. A charge-coupled device (CCD) was used to image the sample and determine where the lasers were focused. The experiments were all performed at room temperature in air, with the exception of a control test conducted in vacuum.

The photonic crystal cavities were tuned by simultaneously irradiating the sample with two lasers through the objective: the $390 \mathrm{~nm}$ ultraviolet (UV) tuning laser, and an $830 \mathrm{~nm}$ nearinfrared pump laser to produce photoluminescence (PL) from the embedded quantum dots.

For the tuning laser, we used a frequency-doubled pulsed Ti:Sapphire laser, producing an output wavelength of $390 \mathrm{~nm}$, pulse repetition frequency of $80 \mathrm{MHz}$, and an average power of $10 \mu \mathrm{W}$ before the microscope objective. The pulse length was approximately $10 \mathrm{ps}$ after passing through a single-mode fiber (SMF) to clean up the beam profile. The UV laser was focused either directly on or immediately adjacent to the cavity to be tuned. The spot size of the tuning laser was roughly $500-700 \mathrm{~nm}$ as estimated from atomic-force microscopy (AFM) scans of photo-oxidized spots.

An $830 \mathrm{~nm}, 350 \mu \mathrm{W}$ SMF-coupled continuous-wave multimode diode laser was used as the PL excitation laser. The PL laser was defocused in order to tightly focus the UV tuning laser, so obtaining a bright PL signal required using a high excitation power. Due to the Purcell effect, the spontaneous emission rate from a photonic crystal cavity is strongly enhanced at its resonant frequencies [22]. The photoluminescence spectrum was thus used to continuously monitor the cavity resonance during the tuning process. In principle, cross-polarized reflectivity could also be used to monitor the cavity resonance when tuning non-photoluminescent devices [3].

\section{Experimental results}

In Fig. 2(a), we present the tuning profile of a single L3 cavity. The tuning rate decreases as a function of time, suggesting a self-limiting mechanism. The tuning rate can be increased by increasing the UV laser power, but using excessively high power risks causing thermal damage. The initial and final spectra are plotted in Fig. 2(b). During the tuning process, the cavity quality factor was degraded from $Q_{\text {initial }}=2990$ to $Q_{\text {final }}=2100$ and the brightness significantly increased, most likely due to scattering from the photo-oxidized spot.

Next, in Figs. 2(c) and 2(d) we present the tuning of a single cavity in a proximity-coupled pair of L3 cavities. The behaviour of such a system can be accurately described using coupledmode theory [23]. Due to the coupling between the cavities, such a system will present two resonant peaks with frequencies $\Omega_{1}, \Omega_{2}$ given by

$$
\Omega_{1,2}=\frac{1}{2}\left(\omega_{1}+\omega_{2}\right) \pm \frac{1}{2} \sqrt{\left(\omega_{1}-\omega_{2}\right)^{2}+J^{2}}
$$

where $\omega_{1}, \omega_{2}$ are the individual cavity frequencies, and $J$ is the coupling between the cavities. We have assumed the cavities are in the strong coupling limit $J \gg \omega_{i} / Q_{i}$, where the $Q_{i}$ is the quality factor of cavity $i$.

The UV laser was focused on one edge of a cavity, as can be seen in an SEM of the tuned structure in Fig. 3(c). As the UV laser was applied, the resonant peak at $1298.5 \mathrm{~nm}$ remained 

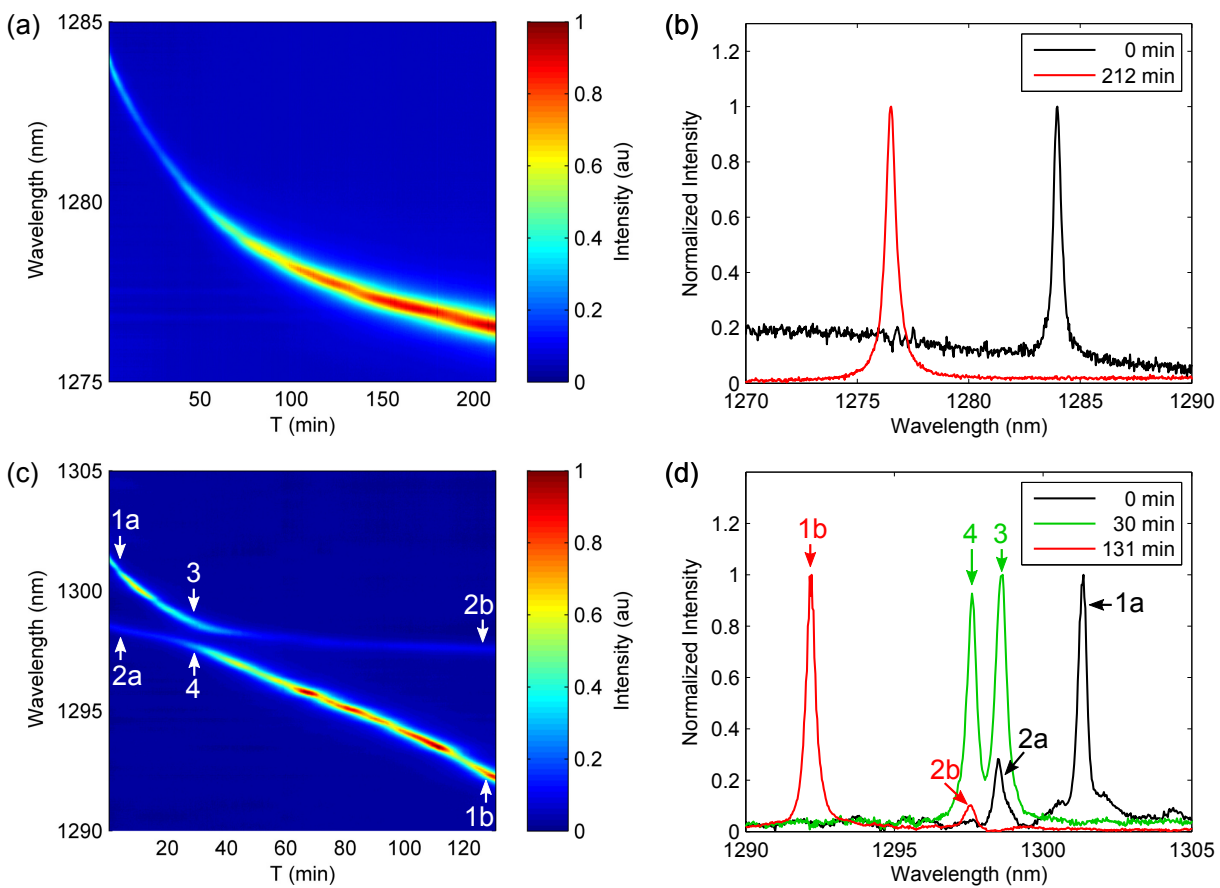

Fig. 2. Photoluminescence spectrum of (a,b) a single L3 cavity and (c,d) coupled L3 cavities as a function of time while tuning with the $390 \mathrm{~nm}$ UV laser. Due to Purcell enhancement, the cavity resonances are clearly visible. We have performed background subtraction, and individually normalized the spectra in (b, d) for clarity. (a) The single cavity was blueshifted by $7.8 \mathrm{~nm}$ during the tuning process. (b) Initial and final photoluminescence spectra for the same cavity. The cavity quality factor was somewhat degraded by the tuning process, being reduced from $Q_{\text {initial }}=2990$ to $Q_{\text {final }}=2100$. (c) In the coupled-cavity system, one cavity was tuned by $9.1 \mathrm{~nm}$, and the other cavity was tuned by only $1.0 \mathrm{~nm}$, resulting in a clear anti-crossing where their resonances became degenerate. The experimentally measured coupling strength $J$ was $0.98 \mathrm{~nm}$, close to the simulated value of $1.2 \mathrm{~nm}$. (d) Initial (0 min), intermediate (30 $\mathrm{min})$, and final $(131 \mathrm{~min})$ spectra for the coupled cavity system. The spectrometer was aligned with the tuned cavity, resulting in a brighter PL signal from the tuned cavity than the untuned cavity through most of the tuning process. At the anti-crossing, the two resonances have equal intensities: both the symmetric and antisymmetric modes split energy evenly between the two cavities [23]. 

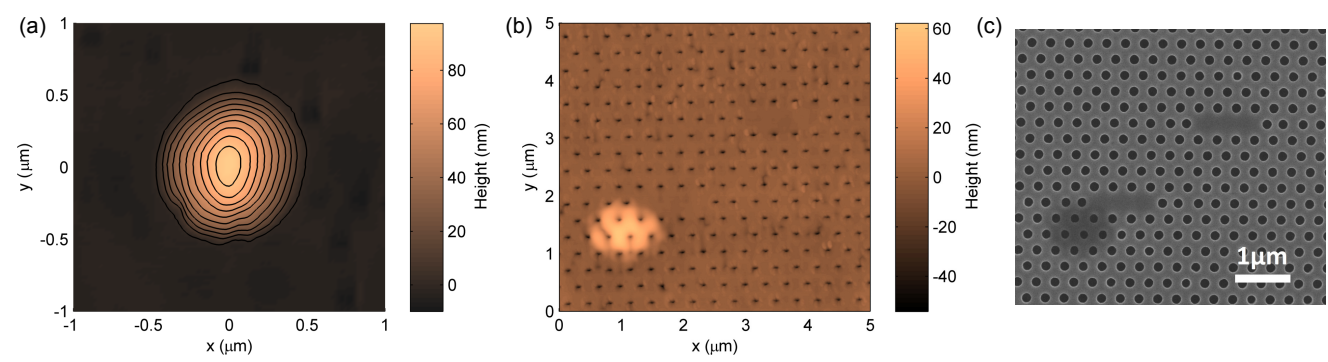

Fig. 3. (a) Non-contact atomic-force microscopy (nc-AFM) scan of a photo-oxidized spot on an unpatterned GaAs membrane. The spot was oxidized using a tightly-focused $10 \mu \mathrm{W}$ spot for $120 \mathrm{~min}$, the same parameters as used to tune the coupled system in Fig. 2(c). The growth of oxide produced a $101.6 \pm 1.0 \mathrm{~nm}$ tall bump with a full-width half maximum (FWHM) of $628 \pm 60 \mathrm{~nm}$. The contour interval is $10 \mathrm{~nm}$. (b) nc-AFM and (c) scanning electron microscopy (SEM) scans of the coupled L3 system tuned in Fig. 2(c), taken after performing tuning. The photonic crystal holes appear to be conical in the AFM scan due to the shape of the AFM tip. The oxidation is visible as a slight discoloration and reduction in hole size in the SEM image.

nearly stationary while the other resonant peak blueshifted from $1301.3 \mathrm{~nm}$ to $1292.2 \mathrm{~nm}$. As the two peaks pass each other, a clear anti-crossing - which is described by Eq. (1) - can be observed.

The tuning mechanism is likely photo-induced oxidation of GaAs by the $390 \mathrm{~nm}$ UV laser in a small $(<1 \mu \mathrm{m})$ spot, resulting in reduction of the local index of refraction and blueshifting the cavities. Previous research has shown that UV light can induce photo-oxidation of GaAs surfaces under similar parameters to our experiment, and the oxide growth rate strongly increases with photon energy [24-26]. In Fig. 3(a), we present an AFM scan of an unpatterned GaAs membrane which was photo-oxidized using the $390 \mathrm{~nm}$ laser. A $101.6 \pm 1.0 \mathrm{~nm}$ tall bump with a full-width half maximum (FWHM) of $628 \pm 60 \mathrm{~nm}$ was formed after irradiating for $120 \mathrm{~min}$ with $10 \mu \mathrm{W}$ of power, probably due to the growth of oxide. AFM and SEM scans of the cavity tuned in Fig. 2(c) are shown in Figs. 3(b) and 3(c), demonstrating the well-localized growth of oxide. Finally, we conducted identical experiments in a vacuum chamber pumped down to $\sim 10^{-4}$ mbar, and there was no observable tuning or change in appearance.

Due to the low power of our UV tuning laser, the tuning mechanism is unlikely to be thermal oxidation. Based on Sentaurus simulations of similar structures, we expect to see a steady-state temperature rise of $<1 \mathrm{~K}$ for a $10 \mu \mathrm{W}$ heat source [27].

The instantaneous temperature rise from each UV laser pulse is expected to be much higher, but still relatively low. Assuming a plane wave propagating into an infinite block of GaAs, and ignoring reflections, the local temperature rise $\Delta T$ from a single pulse is given by

$$
\Delta T=\Phi_{0} \frac{\alpha e^{-\alpha z}}{\rho C}
$$

where $\Phi_{0}$ is the incident fluence $\left(\mathrm{W} / \mathrm{cm}^{2}\right), \alpha=7.433 \times 10^{5} 1 / \mathrm{cm}$ is the extinction coefficient of GaAs at $390 \mathrm{~nm}$ [28], $C=0.350 \mathrm{~J} / \mathrm{g} \mathrm{K}$ and $\rho=5.320 \mathrm{~g} / \mathrm{cm}^{3}$ are the heat capacity and density of GaAs [29], and $z$ is the distance from the incident surface. If we assume the incident light is a gaussian beam with a FWHM of $300 \mathrm{~nm}$, the estimated temperature rise at the surface $(z=0)$ is $48.9 \mathrm{~K}$, far too low for thermally-induced oxidation. This is most likely an overestimate since we assumed there are no reflections and used a conservative estimate of laser spot size. 


\section{Conclusion}

In conclusion, we have demonstrated a technique for tuning GaAs nanophotonic resonators which requires only a low-power UV laser at room temperature in ambient atmosphere. In particular, this technique can be used to independently tune individual cavities in coupledcavity configurations, enabling a wide variety of quantum computing and quantum simulation devices based on cavity networks.

\section{Acknowledgments}

The authors acknowledge support provided by the Air Force Office of Scientific Research (AFOSR) MURI Center for Multi-Functional Light-Matter Interfaces based on Atoms and Solids (FA9550-09-1-0704), and the AFOSR MURI for Complex and Robust On-chip Nanophotonics (FA9550-12-1-0025). AYP acknowledges support from the Stanford Graduate Fellowship. KGL acknowledges support from the Swiss National Science Foundation. 